\title{
Developing Embedded Multi-threaded Applications with CATAPULTS, a Domain-specific Language for Generating Thread Schedulers*
}

\author{
Matthew D. Roper and Ronald A. Olsson \\ Department of Computer Science \\ University of California, Davis \\ Davis, CA 95616-8562 USA \\ \{roper,olsson\}@cs.ucdavis.edu
}

May 19, 2005

\begin{abstract}
This paper describes CATAPULTS, a domain-specific language for creating and testing application-specific user level thread schedulers. Using a domain-specific language to write thread schedulers provides three advantages. First, it modularizes the thread scheduler, making it easy to plug in and experiment with different schedulers. Second, using a domain-specific language for scheduling code helps prevent several of the common programming mistakes that are easy to make when programming in low-level C or assembly. Finally, the CATAPULTS translator has multiple backends that generate code for different languages and libraries. This makes it easy to prototype an embedded application on a regular PC, and then develop the final version on the embedded hardware; the CATAPULTS translator will take care of generating the appropriate code for both the PC prototype and the final embedded version of the program. Using our implementation of CATAPULTS for Z-World's embedded Rabbit processors, we obtained a performance gain of about $12.6 \%$ at the expense of about $12.7 \%$ increase in code size for a fairly typical embedded application.
\end{abstract}

${ }^{*}$ This work is partially supported by Z-World, Inc. and the University of California under the MICRO program. The National Science Foundation partially supported our equipment through grant EIA-0224469. 


\section{Introduction}

Embedded control systems are generally responsible for handling several concurrent tasks (e.g., driving different pieces of hardware) and thus lend themselves to a multi-threaded design. This model is intuitive to program in because it allows each task to be programmed in relative isolation and makes it easy to follow the flow of control inside the task. Threads can either be scheduled cooperatively, where each thread has control of the processor until it explicitly yields it, or preemptively, where context switches are triggered at regular intervals by a timer interrupt. Regardless of which type of threading is used, the algorithm used to schedule threads can have a significant impact on the overall performance of the system. With the limited resources available on an embedded system, the overhead of inefficient context switching is much more noticeable than it would be on regular computer, which has much more processing power.

This paper introduces CATAPULTS, a system for developing application-specific schedulers, and shows how to use CATAPULTS for embedded systems applications. Generating specialized schedulers for a specific application improves performance not only by minimizing inappropriate context switches, but also by speeding up the scheduling algorithm itself; i.e., information such as thread priority or number of activations should only be tracked and processed if an application actually needs it to make good scheduling decisions. Unnecessary bookkeeping can be eliminated.

Our approach uses a domain-specific language for writing application-specific schedulers. It provides three major benefits. First, all scheduling code is collected into a single, replaceable component. The programmer need only fill in the body of various scheduling events (e.g., "new thread," "quantum expired," "thread terminated," etc.) in an aspect-oriented manner. Second, using a domain-specific language allows much better static analysis to be performed than if the scheduler were directly written in low-level $\mathrm{C}$ or assembly language (as most embedded applications are). For example, it is impossible to "lose" a reference 
to a thread using our language. Finally, using a domain-specific language allows multiple translation backends to be developed in order to target different threading libraries or programming languages; this is especially useful when simulating the system on a regular PC before developing the actual embedded version.

Our primary targets for CATAPULTS are small, resource-constrained embedded controllers with low processing power. Such systems generally run the control software directly on the hardware, without the support of a real time operating system. Although we are currently working on extensions to CATAPULTS to aid in the development and verification of soft realtime schedulers, these are not a primary focus of our current implementation.

The rest of this paper is organized as follows. Section 2 provides an introductory example of a CATAPULTS scheduler for a representative embedded application. Section 3 gives an overview of related work. Section 4 describes the purpose and design of the CATAPULTS system. Section 5 provides details on the organization of the CATAPULTS domain-specific language. Section 6 describes how the components of the system were implemented. Section 7 discusses our experience, including performance results, of using CATAPULTS on representative embedded applications. Finally, Section 8 provides some discussion, describes possible avenues for future exploration, and concludes the paper.

\section{An Introductory Example}

CATAPULTS is most easily introduced by providing an example of applying it to a simple, hypothetical multi-threaded application: the embedded control system of a weather monitoring station. The application has to monitor several temperature sensors (which have to be checked with different frequencies), drive a display that changes when the temperature reaches a certain threshold, and perform various calculations while the hardware is idle. Such a situation is relatively easy to model in a multi-threaded application: one thread is assigned to each temperature sensor, one thread drives the output display, and one or more 
threads perform miscellaneous calculations during the processor's idle time.

Control systems of this form are common applications for languages on embedded systems such as Dynamic C [12], an extended subset of C that runs on Z-World's 8-bit Rabbit processors (Dynamic C and our implementation of CATAPULTS on it are discussed in Section 6.2). Although straightforward to implement, a standard Dynamic C implementation as described would fail to utilize the processor fully because Dynamic C's native thread scheduler uses a simple first come, first serve algorithm. Even though some threads do not need to run as often as other threads or only really need to run when certain application-level conditions occur, the Dynamic C scheduler has no such knowledge. It schedules the threads in an inefficient manner, resulting in unnecessary context switches and additional overhead. In our weather monitoring example, the "slow" sensors will be queried for information as often as the "fast" sensors, even though they won't be ready to report information each time.

Using CATAPULTS can make such an application more efficient. It allows the programmer to quickly and easily create a thread scheduler tailored specifically for this application. Figures 1 through 8 show the scheduler specification (some minor details are omitted to save space).

Our example scheduler begins with a thread definition section, shown in Figure 1. It specifies what attributes the scheduler should track for each thread. In this example, only a single attribute ("state") is declared to track the status of a thread (i.e., whether the thread is new, running, suspended, blocked on I/O, etc.).

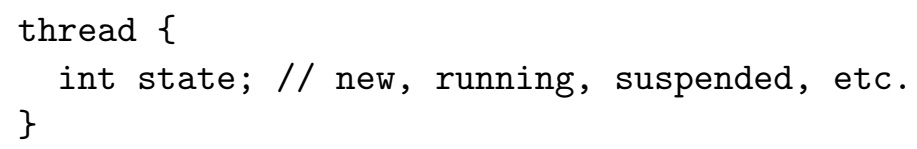

Figure 1: Thread attribute declarations

Next, the scheduler declares which per-thread application variables should be imported into the scheduler. Importing an application variable into the scheduler allows the scheduler 
to monitor the variable for changes made by the application and also allows the scheduler to modify the variable's contents (which is a useful way of communicating information back to the application). Per-thread variables imported this way can be referenced exactly like regular thread attributes in event handler code. Application variable imports are discussed in depth in Section 5.2. Figure 2 illustrates the per-thread imports for our example; a single application variable ("threadclass") is imported, which allows the scheduler to determine the scheduling class ("slow sensor", "display", etc.) to which a given thread belongs.

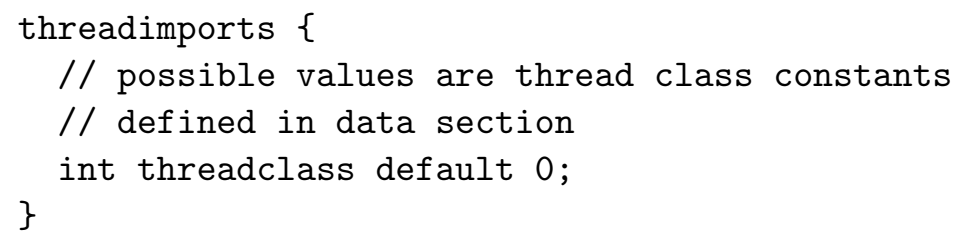

Figure 2: Thread import declarations

The scheduler specification must also include declarations for any global objects used by the scheduler, including both global variables and constants of primitive types (i.e., integers and floats) and thread collections (queues, stacks, etc., provided by the runtime system; see Section 5.1).

Figure 3 shows this data declaration section for our example scheduler. Several thread collections are declared to hold different classes of threads: new (i.e., just created) threads are placed on a stack, sensor threads are divided depending on their speed between two queues, a thread reference is used to hold the single thread that drives the display, and another queue is used to hold the calculation threads. Regular variables of primitive types (just integers in this case) are also defined here to keep track of the last time a thread of a specific class ran, and constants are defined for the different scheduling classes to which a thread can belong.

Just as a scheduler may need to import thread-specific attributes from the application, it may also need to monitor or update regular (global) application variables. For CATAPULTS to link a general application variable with an identifier in the scheduler, the 


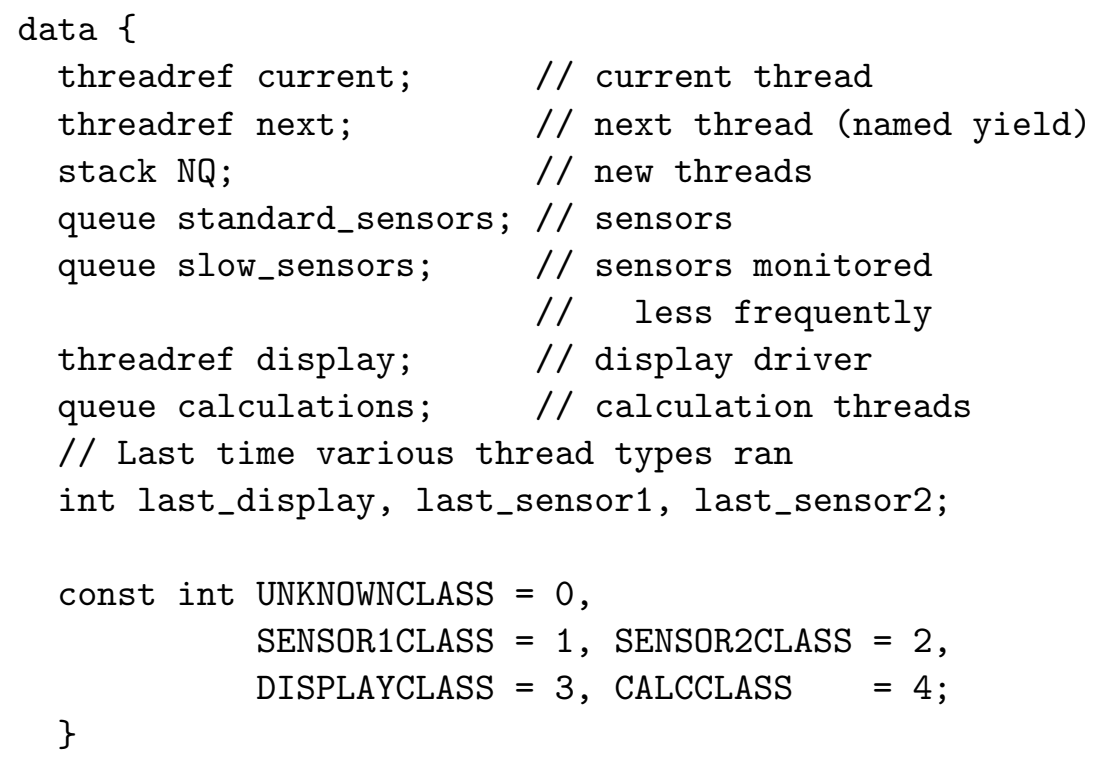

Figure 3: Global data declarations

imported variable must be declared in an imports block, along with a default value to use in case the application does not register the variable or the variable needs to be used by the scheduler before the application has a chance to register it. In our example, a single global variable ("temperature") is imported from the application. This variable will be used later, in the scheduler's event handlers, to determine whether or not the display output thread should be run.

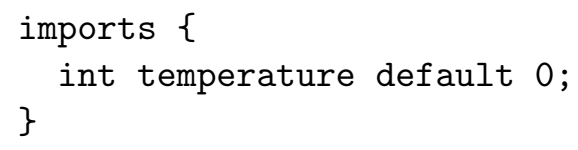

Figure 4: Application variable imports

The remainder of the scheduler definition consists of event and query handlers. These handlers, which resemble $\mathrm{C}$ functions, are callbacks that the base threading library has been modified to call when it needs to perform a scheduling action or get information from the scheduler (see Section 6.2). The difference between an event handler and a query handler is the type of action performed. Event handlers are used when the base threading library is directing the scheduler to perform a specific action (e.g., "suspend this thread"). Event 
handlers are intended to perform side effects by manipulating the scheduler's global data structures; they return no value. In contrast, query handlers are used when the internals of the base threading library need to know something about the scheduler (e.g., "how many threads are currently in the system?"); query handlers return a value and must not have any side effects. Figures 5-8 contain a subset of the example scheduler's event and query handlers (the full set of event and query handlers is not reproduced here to save space).

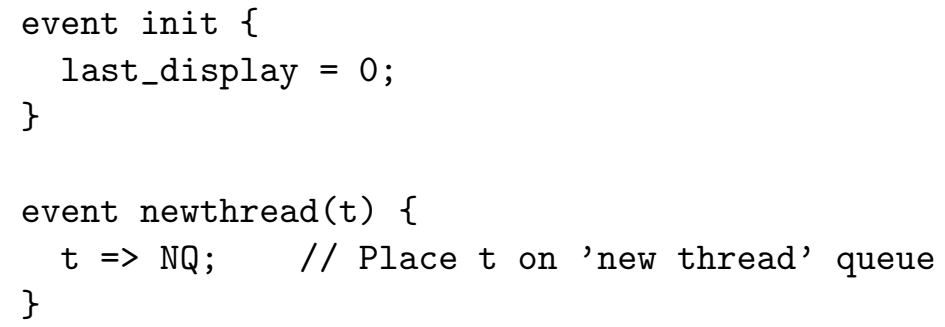

Figure 5: Event handlers to initialize the scheduler and handle new thread creation events

After writing an entire specification, such as that in Figures 1 through 8, the developer then runs the CATAPULTS translator on the specification. It produces a scheduler targeted for a particular backend. The developer then links that scheduler together with the application code.

If the developer decided to prototype/simulate the system on a regular PC before actually developing the embedded Dynamic C version, the scheduler specification could be passed through a different CATAPULTS backend to generate scheduling code for whatever language and library was being used for the prototype.

\section{Related Work}

Very little work has been done in the area of domain-specific languages for writing schedulers. The most closely related project is Bossa [2], a system for generating Linux kernel schedulers using a domain-specific language. Although Bossa is similar in nature to CATAPULTS, it aims to solve a different set of problems. Since Bossa deals with operating system 


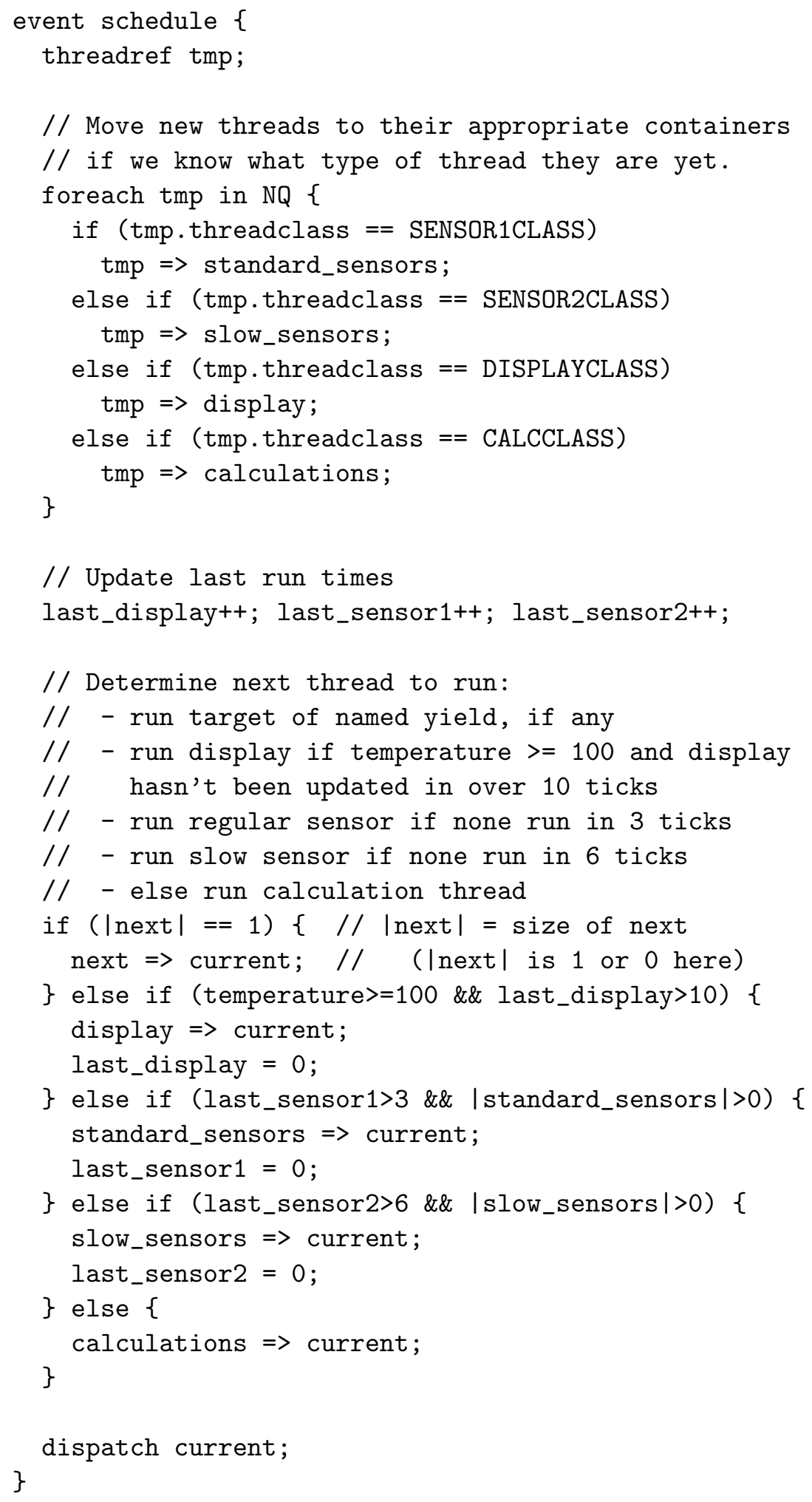

Figure 6: The main scheduling event handler 


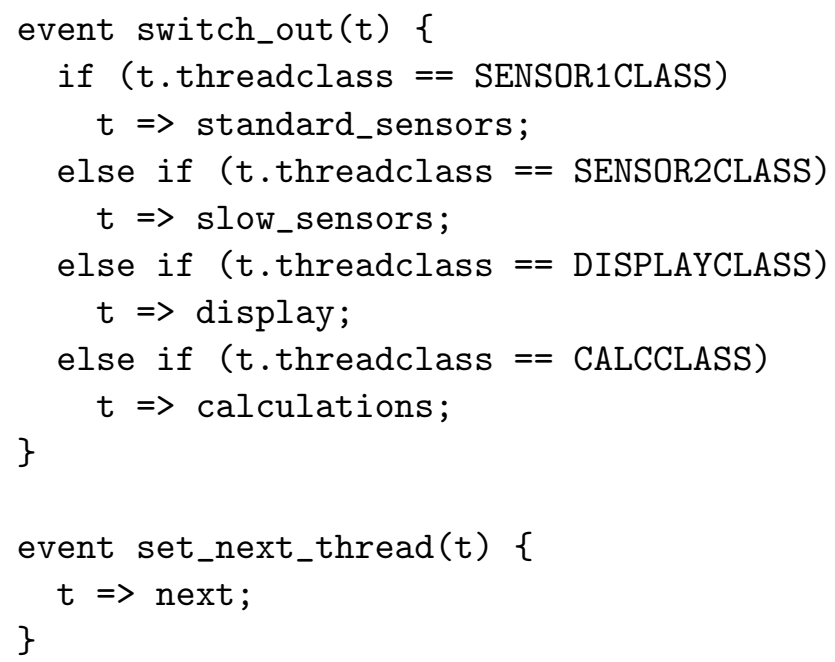

Figure 7: Event handlers for context switching away from a thread and performing a named yield to a specific thread

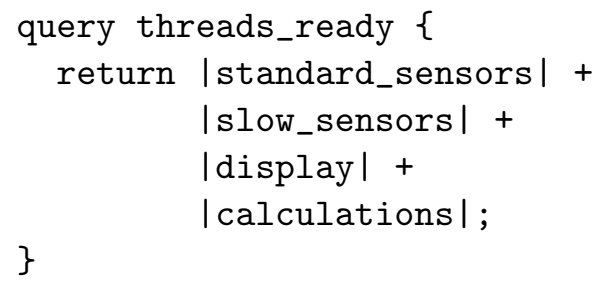

Figure 8: An example query handler that returns to the base threading library the number of threads currently ready to run in the system

schedulers instead of application-level schedulers, its primary focus is on safety rather than performance or expressibility. In Bossa, all operations are guaranteed to be safe, but this limits the overall power of the language. For example, Bossa does not allow any form of unbounded loop; in contrast, CATAPULTS provides traditional for, while, and do loops for cases where a safer foreach loop does not suffice. Our compiler will generate a warning if it cannot be sure that the loop will terminate. CATAPULTS also differs from Bossa in that Bossa is tightly coupled with a specific target language and platform (i.e., it generates Linux kernel $\mathrm{C}$ code). CATAPULTS allows different backends to be written for different target platforms and languages.

Modularizing scheduling code has also started to receive some attention from Linux 
kernel developers. A recent Linux kernel patch [7] separates all scheduling logic out into a separate kernel source file, thus making it much easier to replace the kernel scheduler. Although it appears that this pluggable scheduler framework is unlikely to be accepted into the mainline kernel, it has received notable support and is being developed as an external patch to the kernel. This pluggable scheduler framework provides some of the benefits that systems such as Bossa or CATAPULTS do - modularization and ease of replacement but lacks the portability and safety benefits that can be obtained from using a domainspecific language like CATAPULTS. Had it existed early enough, the pluggable scheduler framework would have been an excellent foundation on which to build Bossa or other kernel-based frameworks.

Other applications of domain-specific languages for embedded systems include Hume [5]. Hume aims to provide a language for programming embedded systems that includes highlevel features such as automatic memory management, exception handling, and polymorphic types, while guaranteeing application resource usage and timing behavior. Hume is intended for actual application development and although threads are provided, their scheduling cannot be changed from the builtin round-robin algorithm.

\section{Design}

Allowing application programmers to replace the thread scheduler, a very highly tuned component of most software systems, is a controversial approach. Although errors introduced in the scheduling specification can result in poor performance or instability, well-written schedulers can result in significantly improved performance. The use of CATAPULTS is an optimization with a tradeoff: higher performance at the cost of additional work writing a CATAPULTS scheduler and less assurance of stability. We expect applications to be written without regards for the scheduler, and then, if higher thread performance is necessary, an application-specific scheduler can be written and plugged-in. The most "dan- 
gerous" feature of CATAPULTS is the use of imported application variables (discussed in Section 5.2) since it allows direct interaction between the application and the scheduler. Importing application variables is an optional feature that allows more specialized scheduler development at the cost of tighter coupling between the application and scheduler; the application developer can decide whether this tradeoff is worthwhile for the specific application. Even if application-specific schedulers that import application variables are not desired, performance can often be enhanced simply by selecting an appropriate generic scheduler for the application. In this case, the scheduler can be developed and fine-tuned by a third party, which makes the use of a CATAPULTS scheduler just as safe and easy as using the original, built-in scheduler.

The CATAPULTS scheduling language was designed with three major goals in mind: modularization and pluggability of scheduling logic, prevention of common programming errors encountered in schedulers, and portability across different scheduling libraries with different capabilities. This section discusses these three goals. A fourth important goal, good performance, is implicit in the design decisions made for CATAPULTS and is discussed in Section 7.

\subsection{Modularization}

Although threading libraries on embedded systems are generally far simpler than their PC counterparts, modifying or replacing the scheduling algorithm used by a thread library is still a non-trivial task. Although it may be easy to locate the function that picks the next thread, most threading libraries have other scheduling code spread throughout the library (e.g., code that manipulates queues of threads when various events occur). Tracking down every such reference to the scheduler's data structures is tedious and error-prone. For example, although the GNU Pth threading library [4] isolates its main scheduling routine in a file pth_sched.c, making any large changes to the Pth scheduler (such as replacing Pth's new thread queue, ready queue, etc. with different data structures and 
thread organizations) can require code modifications to more than 20 files. Furthermore, if the developer wishes to actually change the data structures used to store threads (e.g., add a new queue for threads of a specific type), the modifications required become even more invasive.

With CATAPULTS we overcome this problem by allowing the developer to write the scheduler specification independently from the rest of the threading library. The CATAPULTS translator will then weave the user-specified scheduling code into the rest of the threading library to create a version of the library that is specialized for the specific application. Thus, it is very easy to try out different scheduling strategies.

\subsection{Error Prevention}

Threading libraries for embedded systems are often written in $\mathrm{C}$ or low-level assembly since these are the languages that are most often used for application development. Although $\mathrm{C}$ and assembly are very powerful languages, they do very little to prevent programming errors, especially when manipulating complex data structures via pointers. When such errors occur in thread schedulers, they often take the form of a thread coexisting on two different data structures at once (essentially duplicating a thread) or of a thread's reference being "lost" by the scheduler. Even in higher-level languages these types of mistakes are often easy to make and they are often very hard to track down and debug since the exact scheduling conditions that trigger the bug may not be easy to reproduce.

CATAPULTS provides a very simple (yet seemingly sufficient for most schedulers in our experience) set of data structures for storing collections of threads: threadrefs, stacks, queues, and lists that are sortable on any thread attribute (e.g., priority). All of these containers are unbounded except for threadrefs, which are bounded containers with a single slot. For convenience, individual threadrefs can be grouped into arrays, but each element of the array must be accessed directly; the array itself is not considered to be a thread collection. CATAPULTS enforces the invariant that each thread in the system is contained 
in exactly one collection at any time; this is a strength of CATAPULTS because thread references can never be duplicated or lost due to programmer error (although they can be explicitly destroyed when no longer needed). The only way to add or remove a thread from a container is to use the thread transfer operator, whose syntax is $\operatorname{src}=>$ dest; Each type of thread container has predefined logic that specifies how threads are inserted and removed by the transfer statement (e.g., removal occurs at the end of queues, but at the beginning of stacks). When this transfer operator is encountered in a scheduler specification, the CATAPULTS translator attempts to verify statically that there will be at least one element in the source container; if this cannot be guaranteed, the CATAPULTS translator inserts runtime assertions into the generated code. Similar checks are made to ensure that a bare thread reference used as the destination of a transfer statement does not already contain a thread. All thread transfer operations fall into one of four cases and cause the following types of checks to be performed:

threadref $=>$ threadref Attempt to statically determine that the source threadref is full and that the target threadref is empty. If either of these cannot be determined with certainty, runtime assertions are inserted into the generated code.

threadref $=>$ unbounded container Attempt to statically determine that the source threadref is full. If unable to determine statically, a runtime assertion is inserted into the generated code.

unbounded container $=>$ threadref Attempt to statically determine that the source container contains at least one thread and that the target threadref is empty. If either of these cannot be determined with certainty, runtime assertions are inserted into the generated code.

unbounded container $=>$ unbounded container Attempt to statically determine that the source container contains at least one thread. If unable to determine statically, a runtime assertion is inserted into the generated code. 
It should be noted that due to CATAPULTS' use of callback-like event and query handlers, the empty or full status of a container can only be inferred intra-handler and not interhandler. Since event handlers can be called in any order, the contents of all containers (with the exception of threadrefs used as parameters to a handler) are completely unknown at the start of an event handler. As thread transfers are performed, it will become statically apparent that some containers are not empty (i.e., they have had threads transferred into them) or that some threadrefs are definitely empty (they have had a thread transferred out of them). So in general, less than half of this kind of container checks can be done statically at compile time - only when a container has been previously operated on by the current event or query handler can any information about its contents be inferred.

\subsection{Portability}

One of the primary goals of CATAPULTS is to make it as portable and retargettable as possible. CATAPULTS is not restricted to generating code for any one threading library; different code generation modules can be plugged in to allow generation of scheduling code for different libraries or even different languages. At the moment we have backend code generators for Dynamic C (a C-like language with threading features that is used to program ZWorld's embedded Rabbit controllers [12]) and GNU Pth [4] (a powerful cooperative threading library for PC's); writing more backends for other languages will be straightforward.

The fact that CATAPULTS can compile to different target languages and libraries and has backends for both embedded systems and regular PC's is especially advantageous when simulating or prototyping a system on a PC and then re-writing a final version on the actual embedded hardware. CATAPULTS allows the programmer to develop a scheduler once and then (assuming a CATAPULTS code generation module exists for both languages), simply recompile to generate schedulers for both the prototype and final system, even though they are using different languages and threading libraries. 
Ideally, CATAPULTS would be able recompile schedulers for different threading packages with no modifications to the scheduler specification at all. However, since CATAPULTS allows programmers to write callback routines for various scheduling events, it may be necessary to add code to the scheduler specification when switching to a more featureful output module. For example, a scheduler developed for use with Dynamic C need only specify callback code for a basic set of thread events (thread creation, thread selection, etc.). If that scheduler specification is then used to generate a scheduler for a more advanced threading library, such as Pth, additional code will need to be written to specify what actions to perform on Pth's more advanced scheduling events (e.g., OS signal received, I/O operation complete, etc.). Each CATAPULTS backend code generation module includes a list of the scheduling events that are must be specified in order to create a complete scheduler; if a code generation module is used with a scheduler specification that does not include one or more of the required events, an error will be returned and translation will stop.

\section{$5 \quad$ Language Details}

\subsection{Data Types}

CATAPULTS provides a typical set of primitive types. In addition, CATAPULTS provides several thread container types for organizing the threads in the system: queue, stack, pqueue, pstack, and threadref. A pqueue (or pstack) is similar to a queue (or stack), but its threads are ordered by a user-specified key. A threadref can hold at most one thread. As mentioned in Section 4.2, all threads must be present in one and only one of the scheduler's thread containers at any time and the thread transfer operator is used to move threads between containers. 


\subsection{Imported Application Variables}

As seen in Section 2, CATAPULTS allows two types of variables to be registered (imported) with the scheduler: general (global) application variables and per-thread instance variables. Registering general variables is useful for providing the scheduler with information about the status or load of the system as a whole; common examples include the number of open network connections in a multithreaded Internet server or the number of calculations completed in a scientific application. In contrast, registering per-thread instance variables with the scheduler is useful for tracking information that the application stores for each thread. Per-thread instance variables are useful not only for monitoring information that the application would be tracking anyway (e.g., the number of packets that have been processed on a network connection for an Internet server), but also for specifically directing scheduler behavior from the application, e.g., threadclass declared in Figure 2 and used in Figures 6 and 7.

Imported application variables are the most controversial feature of CATAPULTS since mixing application-level data with scheduler logic can be seen as a dangerous entanglement of separate system levels. This optional feature provides a tradeoff to the application programmer: it becomes harder to change the application without also making changes to the scheduler, but performance can be significantly improved by making use of applicationlevel information.

Although registering variables requires some modification to the base application and removes the transparency of CATAPULTS, the modifications required are minimal; only a single registration statement is necessary near the beginning of the program for each variable that is to be registered with the scheduler. 


\subsection{Verbatim Statements and Expressions}

CATAPULTS provides verbatim statements and expressions, which allow the programmer to include a block of code (either a statement-level block or a single expression) of the target language directly in the scheduler. For example, a scheduler that uses a random number generator to make some of its scheduling decisions will use verbatim statements to generate random numbers because CATAPULTS has no instructions to do so. Thus, the programmer can express anything that could be coded in the target scheduler's programming language at the expense of some portability (i.e., the verbatim statements and expressions will need to be re-written for each target language/library to which the scheduler is compiled).

\section{Implementation}

This section describes two key parts of the CATAPULTS implementation: its frontend and one of its backends. As noted earlier, CATAPULTS supports multiple backend code generators. Each is written as a separate module, which makes it easy to add new backends for other languages and libraries. We have currently implemented two such code generation modules for CATAPULTS: a Dynamic C backend (for embedded systems) and a GNU Pth backend (for PC's). Below, we focus on the Dynamic C backend; Reference [11] discusses the Pth backend.

\subsection{The Frontend}

The CATAPULTS translator is written in Python using PLY (Python Lex/Yacc) [3]. The translator uses very simple propagation-based static analysis to track the various invariants described in Section 4.2. Specifically, this static analysis is used to track the following information:

- presence or absence of threads on a container or in a thread reference 
- failure to store a thread passed as a parameter into a permanent container in an event handler that requires this (e.g., newthread (t))

- failure of a query handler to return a value

- failure of an event handler to produce a side effect

- code following a dispatch or return statement

- statically known variable values

As discussed in Section 4.2, CATAPULTS generates runtime assertions in the generated code for scheduler code that it cannot analyze statically.

\subsection{The Dynamic C Backend}

To apply CATAPULTS in an embedded environment, we developed a CATAPULTS backend for Dynamic C, an extended subset of C that is used to program Z-World's 8-bit Rabbit devices. Dynamic $\mathrm{C}$ includes builtin cooperative multithreading in the form of costatements and cofunctions, but only allows a round-robin scheduling policy for the threads. Although it is possible to use tricks to accomplish dynamic scheduling in Dynamic C [10], doing so requires invasive changes to the application itself, which results in confusing code and does not integrate well with CATAPULTS. Instead we chose to integrate CATAPULTS with the cmthread.lib threading library that we had previously written for Dynamic C. cmthread.lib is a substitute for Dynamic C's language-level multithreading and provides an API that is more consistent with other popular threading API's such as Pth or Pthreads. cmthread.lib also provides better performance in many cases.

Dynamic $\mathrm{C}$ applications run in an embedded environment with no operating system. Our Dynamic C backend generates a custom version of the cmthread.lib library that contains the custom generated scheduling code inline. The modifications to cmthread.lib to make it work with CATAPULTS are minor: about 100 new lines of code were added to the 
Table 1: Comparison of CATAPULTS threading library and generic cmthread.lib

\begin{tabular}{|l||c|c|c|}
\hline & $\begin{array}{c}\text { Lines of } \\
\text { Code }\end{array}$ & $\begin{array}{c}\text { Compiled } \\
\text { Code Size }\end{array}$ & $\begin{array}{c}\text { Simulation } \\
\text { Duration }\end{array}$ \\
\hline \hline Generic cmthread.lib & 457 & 21120 bytes & $76.508 \mathrm{sec}$ \\
\hline Generated CATAPULTS library & $<546+517$ & 23808 bytes & $66.837 \mathrm{sec}$ \\
\hline
\end{tabular}

original 457 lines. Because this new code is being generated by CATAPULTS, its formatting sometimes splits what would normally be one line of code over several. So, a fairer estimate is about 50 lines of new code. Also, a good portion of this code is functions that simply do callbacks.

(In contrast, our Pth implementation dynamically loads schedulers at runtime [11], which is neither possible nor advantageous in the Dynamic $\mathrm{C}$ environment; it therefore uses indirect function calls via function pointers, which incurs some additional overhead.)

\section{Experience}

Below, we describe some of our experiences using CATAPULTS for embedded systems applications. We have also used CATAPULTS with the Pth backend for regular PC applications such as the CoW web server and numerous small schedulers [11].

\subsection{Weather Monitoring Station Application}

In order to measure the benefit of using CATAPULTS on an embedded application, we simulated the weather monitoring station example described in Section 2. Since we do not have access to real weather monitoring hardware, we wrote a Dynamic $\mathrm{C}$ application with the appropriate control logic and replaced actual sensor and display hardware I/O with small loops. The CATAPULTS scheduler specification described in the example in Section 2 was used to control thread scheduling. The complete specification (including the minor details omitted in Figures 1-8) was a total of 174 lines of code and was translated into 546 lines of 
Dynamic C. In contrast, the original cmthread.lib library on which CATAPULTS' output is based is a total of 457 lines of Dynamic C code. Although space is a scarce resource on embedded systems, this size increase is quite reasonable considering how much more sophisticated the generated scheduler is than the simple first-come, first-serve scheduler in cmthread.lib. The CATAPULTS library also links with another 517 line auxiliary library that contains implementations of the various thread container types provided by CATAPULTS. The Dynamic C compiler will only link in the functions from this auxiliary library that are actually used by the specific application, so only a couple hundred of these lines are likely to be included in any given application. So, comparing only lines of code is somewhat misleading; comparing code size is more useful. After compiling the simulation application along with the threading library, the total code size downloaded to the Rabbit processor was, as shown in Table 1, 23808 bytes when the generated CATAPULTS library was used as compared to 21120 bytes when the generic cmthread.lib was used (i.e., a $12.7 \%$ increase in size).

To measure the performance difference between the CATAPULTS generated scheduler and generic cmthread.lib scheduler, we executed the control simulation until a total of 10000 executions of the "calculation" threads had run and then measured the total runtime. When using the generic cmthread.lib, we allow threads to notice that they have no work to do and yield immediately; this eliminates the additional overhead of useless hardware I/O, but still incurs the overhead of an unnecessary context switch. As shown in Table 1, the simulation completed almost 10 seconds faster when using the CATAPULTS-generated scheduler (a $12.6 \%$ speedup).

\subsection{CoW Web Server}

We also adapted CoW [6], a cooperatively multithreaded web server, to use a CATAPULTS scheduler. The version of CoW that runs on the Rabbits uses the standard Dynamic C first-come, first-serve scheduler. We identified that we could obtain better performance if 
the scheduler was tailored to the specific requirements of CoW. In particular, the more quickly HTTP requests are processed and flushed from the system, the faster new requests can be accepted. By giving greater priority to request handler threads that are near completion, the overall throughput of the system can be significantly increased. The complete CATAPULTS scheduler specification for CoW appears in Reference [9].

\section{Conclusion}

Although real world embedded systems are likely to have a very diverse set of scheduling requirements, we have applied CATAPULTS to what we believe to be a typical embedded system and have achieved very positive results. Performance gains are likely to vary widely depending on the complexity of the scheduling algorithm required by a given system and by the penalty incurred by inefficient thread scheduling, but we have shown that developing custom thread schedulers with CATAPULTS is relatively straightforward and can provide significant performance gains. Moreover, using CATAPULTS provides more safety and portability.

We have a number of plans for future exploration. We realize that many schedulers share common code and data structure organization, so we would like to make it possible to derive new schedulers from existing ones and then override specific parts in an objectoriented manner. Hierarchical thread scheduling (e.g., as discussed in Reference [1]) is another area we would like to explore; CATAPULTS could be extended to develop different scheduling algorithms for different subgroups of threads. Such hierarchical schemes would allow different people to develop different schedulers for subgroups of threads in an application and then schedule those subgroups according to a higher-level scheduler. It would also be interesting to explore the possibility of creating schedulers graphically with a GUI design tool; this would be especially attractive to engineers of a mechanical system who do not have a lot of experience with software development. Finally, CATAPULTS 
could be extended to work in a distributed environment with systems like DesCaRTes [8] that distribute threads over a network of embedded, uniprocessor controllers.

\section{Acknowledgments}

Jason Cheung, especially, and Glen Sanford helped with the development and testing of

\section{CATAPULTS.}

\section{References}

[1] D. Auslander, J. Ridgely, and J. Ringgenberg. Control Software for Mechanical Systems: ObjectOriented Design in a Real-Time World. Prentice Hall PTR, 2002.

[2] L. Barreto and G. Muller. Bossa: A language-based approach for the design of real time schedulers. In 10th International Conference on Real-Time Systems (RTS), 2002.

[3] D. Beazley. PLY (Python Lex-Yacc). http://systems.cs.uchicago.edu/ply/.

[4] R. S. Engelschall. GNU Pth - the GNU Portable Threads. http://www.gnu.org/software/pth/.

[5] K. Hammond and G. Michaelson. Hume: a domain-specific language for real-time embedded systems. In Proceedings of the Second International Conference on Generative Programming and Component Engineering, 2003.

[6] T. Ishihara and M. D. Roper. CoW: A cooperative multithreading web server. http://www.cs. ucdavis.edu/ roper/cow/.

[7] C. Kolivas. Pluggable CPU scheduler framework, October 2004. http://groups-beta.google.com/ group/fa.linux.kernel/msg/891f15d63e5f529d.

[8] J. T. Maris, M. D. Roper, and R. A. Olsson. DesCaRTes: a run-time system with SR-like functionality for programming a network of embedded systems. Computer Languages, Systems and Structures, 29(4):75-100, Dec. 2003.

[9] M. D. Roper. CATAPULTS scheduler code for CoW webserver. http://www.cs.ucdavis.edu/ roper/catapults/examples/embcow.sched.

[10] M. D. Roper. Dynamic threading and scheduling with Dynamic C. http://www.cs.ucdavis.edu/ roper/dcdynthread/.

[11] M. D. Roper and R. A. Olsson. CATAPULTS: Creating and testing application-specific user level thread schedulers. in preparation.

[12] Z-World Inc. Dynamic C user's manual. http://www.zworld.com/documentation/docs/manuals/ DC/DCUserManual/index.htm. 